\title{
Transversals, plexes, and multiplexes in iterated quasigroups
}

\author{
Anna Taranenko* \\ Sobolev Institute of Mathematics; \\ Novosibirsk State University \\ Novosibirsk, Russia \\ taa@math.nsc.ru
}

Submitted: Sep 10, 2017; Accepted: Oct 25, 2018; Published: Nov 2, 2018

(C) The author. Released under the CC BY-ND license (International 4.0).

\begin{abstract}
A $d$-ary quasigroup of order $n$ is a $d$-ary operation over a set of cardinality $n$ such that the Cayley table of the operation is a $d$-dimensional latin hypercube of the same order. Given a binary quasigroup $G$, the $d$-iterated quasigroup $G^{[d]}$ is a $d$-ary quasigroup that is a $d$-time composition of $G$ with itself. A $k$-multiplex (a $k$-plex) $K$ in a $d$-dimensional latin hypercube $Q$ of order $n$ or in the corresponding $d$-ary quasigroup is a multiset (a set) of $k n$ entries such that each hyperplane and each symbol of $Q$ is covered by exactly $k$ elements of $K$. It is common to call 1-plexes transversals.

In this paper we prove that there exists a constant $c(G, k)$ such that if a $d$ iterated quasigroup $G$ of order $n$ has a $k$-multiplex then for large $d$ the number of its $k$-multiplexes is asymptotically equal to $c(G, k)\left(\frac{(k n) !}{k ! n}\right)^{d-1}$. As a corollary we obtain that if the number of transversals in the Cayley table of a $d$-iterated quasigroup $G$ of order $n$ is nonzero then asymptotically it is $c(G, 1) n !^{d-1}$.

In addition, we provide limit constants and recurrence formulas for the numbers of transversals in two iterated quasigroups of order 5 , characterize a typical $k$-multiplex and estimate numbers of partial $k$-multiplexes and transversals in $d$ iterated quasigroups.
\end{abstract}

Mathematics Subject Classifications: 05B15, 05D15, 20N05

*Supported by the Moebius Contest Foundation for Young Scientists. 


\section{Definitions and preliminaries}

Let $n, d \in \mathbb{N}, I_{n}^{d}=\left\{\left(\alpha_{1}, \ldots, \alpha_{d}\right): \alpha_{i} \in\{1, \ldots, n\}\right\}$, and let $I_{n}^{1}=\{1, \ldots, n\}$. Denote by $I_{\{n, k\}}$ the multiset of size $k n$ over the set $\{1, \ldots, n\}$ in which each of $n$ symbols appears exactly $k$ times.

A $d$-dimensional matrix $A$ of order $n$ is an array $\left(a_{\alpha}\right)_{\alpha \in I_{n}^{d}}, a_{\alpha} \in \mathbb{R}$. The support of a matrix $A$ is the set of indices at which the matrix has nonzero values.

For $k \in\{0, \ldots, d\}$, let a $k$-dimensional plane in $A$ be a submatrix of $A$ obtained by fixing $d-k$ indices and letting the other $k$ indices vary from 1 to $n$. A 1-dimensional plane is said to be a line, and a $(d-1)$-dimensional plane is a hyperplane.

A d-dimensional latin hypercube $Q$ of order $n$ is a $d$-dimensional matrix of order $n$ filled by $n$ symbols so that all symbols within each line are distinct. 2-dimensional latin hypercubes are known as latin squares.

A d-dimensional permutation matrix $M$ of order $n$ is a multidimensional $(0,1)$-matrix such that each line contains exactly one unity entry.

A d-ary quasigroup $f$ of order $n$ is a function $f: I_{n}^{d} \rightarrow I_{n}^{1}$ such that the equation $x_{0}=f\left(x_{1}, \ldots, x_{d}\right)$ has a unique solution for any one variable if all the other $d$ variables are specified arbitrarily. In particular, a binary quasigroup of order $n$ is a binary operation * over the set $I_{n}^{1}$ with the following property: for each $a_{0}, a_{1}, a_{2} \in I_{n}^{1}$ there exist unique $x_{1}, x_{2} \in I_{n}^{1}$ such that both $a_{0}=a_{1} * x_{2}$ and $a_{0}=x_{1} * a_{2}$ hold.

$d$-ary quasigroups $f$ and $g$ of order $n$ are called isotopic if there exist permutations $\sigma_{i} \in S_{n}, i=0, \ldots, d$ such that

$$
f\left(x_{1}, \ldots, x_{d}\right)=\sigma_{0}^{-1}\left(g\left(\sigma_{1}\left(x_{1}\right), \ldots, \sigma_{d}\left(x_{d}\right)\right)\right) .
$$

There are natural correspondences between permutation matrices, latin hypercubes, and quasigroups. The Cayley table of a $d$-ary quasigroup $f$ of order $n$ is a $d$-dimensional latin hypercube $Q(f)$ of the same order, and vice versa, every $d$-dimensional latin hypercube can be considered as the Cayley table of some $d$-ary quasigroup. The graph $\left\{\left(x_{0}, x_{1}, \ldots, x_{d}\right): x_{0}=f\left(x_{1}, \ldots, x_{d}\right)\right\}$ of a quasigroup $f$ is the set of unity entries of the $(d+1)$-dimensional permutation matrix $M(f)$ of order $n$. The correspondence between a $d$-dimensional latin hypercube $Q$ and the $(d+1)$-dimensional permutation matrix $M(Q)$ is given by the following rule: an entry $q_{\alpha_{1}, \ldots, \alpha_{d}}$ of a latin hypercube $Q$ equals $\alpha_{d+1}$ if and only if an entry $m_{\alpha_{1}, \ldots, \alpha_{d+1}}$ of the permutation matrix $M(Q)$ equals 1 . In particular, the Cayley table of every binary quasigroup $G$ is a latin square $Q(G)$ that corresponds to some 3-dimensional permutation matrix $M(G)$.

Although all these three approaches (via quasigroups, latin hypercubes, and permutation matrices) are equivalent, in different environments it is more customary to use only one of them. During this paper we will change between these three concepts repeatedly because algebraic properties of quasigroups are needed for the proofs, the research is motivated by problems for latin squares and hypercubes, but the approach via multidimensional permutation matrices is often more symmetric and convenient.

Given a binary quasigroup $G$ of order $n$ defined by a binary operation $*$, the $d$-iterated 
quasigroup $G$ denoted by $G^{[d]}$ is the $d$-ary quasigroup of order $n$ such that

$$
x_{0}=G^{[d]}\left(x_{1}, \ldots, x_{d}\right) \Leftrightarrow\left(\cdots\left(\left(x_{0} * x_{1}\right) * x_{2}\right) * \cdots * x_{d-1}\right) * x_{d}=1,
$$

where 1 stands for the (not necessarily identity) element from the ground set $I_{n}^{1}=$ $\{1, \ldots, n\}$ of the binary quasigroup $*$. Note that the quasigroup $G^{[d]}$ can be equivalently defined by the equation

$$
\left(\cdots\left(\left(x_{0} * x_{1}\right) * x_{2}\right) * \cdots * x_{d-2}\right) * x_{d-1}=1 / x_{d},
$$

where / is the right division for the quasigroup $*$. So we have that the quasigroup $G^{[d]}$ is isotopic to the following quasigroup

$$
\left(\cdots\left(\left(x_{0} * x_{1}\right) * x_{2}\right) * \cdots * x_{d-2}\right) * x_{d-1}=x_{d} .
$$

Because isotopy preserves the properties which we will be interested in, we prefer to use the first definition due to its symmetry.

We denote by $Q\left(G^{[d]}\right)$ the Cayley table of the $d$-iterated quasigroup $G$, and the corresponding $(d+1)$-dimensional permutation matrix is denoted by $M\left(G^{[d]}\right)$.

Let $A$ be a $d$-dimensional matrix of order $n$. A multiset $K$ of $k n$ indices $\left\{\alpha^{1}, \ldots, \alpha^{k n}\right\}$ is called a $k$-multidiagonal if each hyperplane of $A$ contains exactly $k$ elements of $K$. A $k$-multidiagonal $K$ is called a $k$-diagonal if all elements of $K$ are different (namely, $K$ is a set).

A diagonal (or a 1-diagonal) in a multidimensional matrix is a set of $n$ indices such that there is exactly one index in each hyperplane. Note that if we unite any $k$ diagonals we obtain a $k$-multidiagonal, and if we unite $k$ mutually disjoint diagonals we get a $k$ diagonal. Meanwhile, not every $k$-multidiagonal or $k$-diagonal can be partitioned into diagonals. The simplest example is the following 2-diagonal in a 3-dimensional matrix of order 2:

$$
(1,1,1) \quad(1,2,2) \quad(2,1,2) \quad(2,2,1)
$$

For a $d$-dimensional $(0,1)$-matrix $A$ of order $n$, define the $k$-permanent $\operatorname{per}_{k} A$ of a matrix $A$ to be the number of positive $k$-diagonals in $A$. Similarly, the $k$-multipermanent $\operatorname{Per}_{k} A$ of $A$ is the number of positive $k$-multidiagonals in the matrix $A$. Introduction of the term " $k$-permanent" for these objects is explained by the fact that the 1-permanent is exactly the permanent of multidimensional matrices that was studied in detail in [7].

Note that for every $d$-dimensional $(0,1)$-matrix $A$ of order $n$ we have

$$
\operatorname{per}_{k} A \leqslant \operatorname{Per}_{k} A \leqslant\left(\frac{(k n) !}{k !^{n}}\right)^{d-1},
$$

because every $k$-diagonal is a $k$-multidiagonal and because if we fix the permutation in one coordinate of a multidiagonal then in each of the other $d-1$ coordinates we have a permutation of the multiset $I_{\{n, k\}}$.

In a $d$-dimensional latin hypercube $Q$, a diagonal whose entries contain all different symbols is said to be a transversal. Let us define a $k$-plex in a $d$-dimensional latin 
hypercube $Q$ of order $n$ as a selection of $k n$ different indices of $Q$ in which each hyperplane and symbol is represented precisely $k$ times. Analogously, we define a $k$-multiplex in a latin hypercube to be a multiset of indices satisfying the same property.

From the definitions it follows that every transversal in a latin hypercube $Q$ corresponds to a positive diagonal in the permutation matrix $M(Q)$, every $k$-plex ( $k$-multiplex) in $Q$ corresponds to a positive $k$-diagonal (a positive $k$-multidiagonal) in $M(Q)$, and conversely. For $d$-ary quasigroups we define transversals, $k$-plexes, and $k$-multiplexes so that they correspond to those in latin hypercubes. Finally, for the sake of brevity and unification, we will call positive $k$-multidiagonals and $k$-diagonals in $(0,1)$-matrices $k$-multiplexes and $k$-plexes, respectively.

Note that the number of transversals in any $d$-dimensional latin hypercube of order $n$ is not greater than $n !^{d-1}$. Also, it is not hard to check that isotopic $d$-ary quasigroups have the same numbers of $k$-plexes and $k$-multiplexes (for proof of this fact for transversals, see $[9])$.

We will say that a $k$-multiplex $K$ is divisible if there exist a $k_{1}$-multiplex $K_{1}$ and a $k_{2}$-multiplex $K_{2}$ such that $K$ is the union of $K_{1}$ and $K_{2}$; otherwise $K$ is called indivisible. A $k$-multiplex $K$ in a $d$-dimensional matrix of order $n$ is disconnected if $K$ is the union of $k$-multiplexes $K_{1}$ and $K_{2}$ in submatrices of orders $n_{1}$ and $n_{2}\left(n=n_{1}+n_{2}\right)$; otherwise $K$ is said to be connected.

For a $k$-multiplex $K=\left\{\alpha^{1}, \ldots, \alpha^{k n}\right\}$ we denote by $K_{j}$ the $k n$-vector $\left(\alpha_{j}^{1}, \ldots, \alpha_{j}^{k n}\right)$, where $\alpha_{j}^{i}$ is the $j$ th component of the index $\alpha^{i}$. Given a binary quasigroup $*$ and two $m$-vectors $U$ and $V$, a component-wise product of $U$ and $V$ is

$$
U * V=W \Leftrightarrow u_{i} * v_{i}=w_{i} \text { for all } i=1, \ldots, m .
$$

A vector with all unity components is denoted by $E$.

\section{Motivation and the main result}

Transversals and plexes in latin squares have been widely studied for the last decades but many important questions on existence are not solved yet. One of the best known conjectures on transversals belongs to Ryser [6].

Conjecture 1 (Ryser). Every latin square of odd order has a transversal.

According to [1], the following conjecture for 2-plexes in latin squares was proposed by Rodney.

Conjecture 2 (Rodney). Every latin square has a 2-plex.

For the Cayley tables of groups this conjecture was proved by Vaughan-Lee and Wanless.

Theorem 3 ([10]). If $G$ is a group then the latin square $Q(G)$ has a 2-plex. 
One of the possible ways to generalize plexes was considered by Pula in [5], and a comprehensive survey of other results on transversals and $k$-plexes in latin squares is given in [11].

Investigation of transversals in latin hypercubes started only recently. In [11] Wanless generalized Ryser's conjecture for latin hypercubes.

Conjecture 4 (Wanless). Every latin hypercube of odd dimension or odd order has a transversal.

It is known that if $n$ and $d$ are both even then the Cayley table of the $d$-iterated group $\mathbb{Z}_{n}$ has no transversals $[7,11]$. Moreover, using the same technique it is easy to prove the analogous statement for $k$-multiplexes for odd $k$.

Proposition 5. Let $n$ and $d$ be even and $k$ be odd. Then the d-dimensional latin hypercube $Q\left(\mathbb{Z}_{n}^{d]}\right)$ has no k-multiplexes.

Proof. Assume that a multiset of indices $K=\left\{\alpha^{1}, \ldots, \alpha^{k n}\right\}$ is a $k$-multiplex in the latin hypercube $Q\left(\mathbb{Z}_{n}^{[d]}\right)$. Consider the sum

$$
S=\sum_{i=1}^{k n} \sum_{j=0}^{d} \alpha_{j}^{i}
$$

Since for each $\alpha^{i} \in K$ it holds $\sum_{j=0}^{d} \alpha_{j}^{i} \equiv 0 \bmod n$, we have $S \equiv 0 \bmod n$. On the other hand, for each $j \in\{0, \ldots, d\}$ we have $\sum_{i=1}^{k n} \alpha_{j}^{i}=k \frac{n(n+1)}{2}$. Therefore,

$$
S=\sum_{j=0}^{d} \sum_{i=1}^{k n} \alpha_{j}^{i}=\sum_{j=0}^{d} k \frac{n(n+1)}{2}=k(d+1) \frac{n(n+1)}{2} \not \equiv 0 \bmod n,
$$

because $n$ and $d$ are even and $k$ is odd.

The numbers of transversals in all latin hypercubes of orders 2 and 3 are found in [7], and the numbers of transversals in $d$-iterated groups of order 4 are calculated in [9]. Also, in [9] it is proved that for all odd $d$ a $d$-iterated quasigroup of order 4 has transversals, and a lower bound on their number is obtained.

The asymptotic behavior of the maximum number of transversals in latin hypercubes of fixed dimension and large order was found in $[3,8]$. In [2] it was proved that if for an abelian group $G$ the latin hypercube $Q\left(G^{[d]}\right)$ has a transversal then for large $n$ and fixed $d$ the number of transversals in $Q\left(G^{[d]}\right)$ asymptotically reaches the upper bound.

One of the main results of this paper is that the analogous statement holds for the Cayley tables of $d$-iterated quasigroups of large dimension. Namely, it will be a special case of the following theorem. 
Theorem 6. Let $G$ be a binary quasigroup of order $n$ and let $M\left(G^{[d-1]}\right)$ be the $d$ dimensional permutation matrix of the $(d-1)$-iterated quasigroup $G$.

1. For all $k$ and for all even $d$ the d-dimensional permutation matrix $M\left(G^{[d-1]}\right)$ has a nonzero k-multipermanent. If for some odd $d^{\prime}$ we have that $\operatorname{Per}_{k} M\left(G^{\left[d^{\prime}-1\right]}\right)$ is positive then $\operatorname{Per}_{k} M\left(G^{[d-1]}\right)$ is greater than zero for all $d \geqslant d^{\prime}$.

2. There exists a constant $0<c(G, k) \leqslant 1$ such that

$$
\lim _{d \rightarrow \infty} \frac{\operatorname{Per}_{k} M\left(G^{[d-1]}\right)}{\left(\frac{(k n) !}{k !^{n}}\right)^{d-2}}=c(G, k),
$$

where $d$ are taken so that $\operatorname{Per}_{k} M\left(G^{[d-1]}\right)$ is nonzero.

Corollary 7. Let $G$ be a binary quasigroup of order $n$ and let $Q\left(G^{[d]}\right)$ be the d-dimensional latin hypercube that is the Cayley table of the d-iterated quasigroup $G$.

1. For all odd $d$ the latin hypercube $Q\left(G^{[d]}\right)$ has a transversal. If for some even $d^{\prime}$ we have that $Q\left(G^{\left[d^{\prime}\right]}\right)$ has transversals then the latin hypercubes $Q\left(G^{[d]}\right)$ have transversals for all $d \geqslant d^{\prime}$.

2. There exists a constant $0<c(G, 1) \leqslant 1$ such that if the latin hypercube $Q\left(G^{[d]}\right)$ has transversals then for large $d$ the number of transversals is asymptotically equal to $c(G, 1) n !^{d-1}$.

\section{Corollaries of the main result}

Before the proof of Theorem 6 we deduce several corollaries. For this purpose, we need the following auxiliary lemmas about plexes, multiplexes, and $k$-multipermanents.

Lemma 8. 1. If $A$ is a d-dimensional $(0,1)$-matrix $A$ of order $n$ and if for some $k$ we have $\operatorname{Per}_{k} A>0$ then for all integers $m \geqslant 1$ it holds $\operatorname{Per}_{k m} A>0$.

2. Let $G$ be a binary quasigroup of order $n$. If for some $k$ and $d$ we have that $\operatorname{Per}_{k} M\left(G^{[d]}\right)>0$ then for all integers $m \geqslant 0$ it holds that $\operatorname{Per}_{k} M\left(G^{[d+2 m]}\right)>0$. Also, it holds that $\operatorname{per}_{k} M\left(G^{[d+2 m]}\right) \geqslant\left(\frac{(k n) !}{k !^{n}}\right)^{m} \operatorname{per}_{k} M\left(G^{[d]}\right)$.

Proof. 1. It follows from the fact that a union of $m$ copies of any $k$-multiplex is a $\mathrm{km}$ multiplex.

2. Let $K=\left\{\alpha^{1}, \ldots, \alpha^{k n}\right\}$ be a $k$-multiplex over the support of $M\left(G^{[d]}\right)$. By the definition of a $k$-multiplex, we have

$$
\left(\cdots\left(\left(K_{0} * K_{1}\right) * K_{2}\right) * \cdots * K_{d-1}\right) * K_{d}=E .
$$

Let $U=\left(u^{1}, \ldots, u^{k n}\right)$ be a $k n$-vector that is a permutation of the multiset $I_{\{n, k\}}$. Define vector $V$ from the relation $((E * U) * V)=E$. 
Since $*$ is the operation of the quasigroup $G$, the vector $V$ is a permutation of the multiset $I_{\{n, k\}}$. Consequently, for each $i$ the index $\beta^{i}=\left(\alpha_{1}^{i}, \ldots, \alpha_{d}^{i}, u^{i}, v^{i}\right)$ belongs to the support of $M\left(G^{[d+2]}\right)$. Therefore, $K^{\prime}=\left\{\beta^{1}, \ldots, \beta^{k n}\right\}$ is a $k$-multiplex in the multidimensional permutation matrix $M\left(G^{[d+2]}\right)$. It only remains to note that if $K$ is a $k$-plex in $M\left(G^{[d]}\right)$ then each choice of $U$ leads to a different $k$-plex $K^{\prime}$ in $M\left(G^{[d+2]}\right)$.

We will say that a $k$-multiplex is a true $k$-multiplex if it is not a $k$-plex.

Lemma 9. Let $A$ be a d-dimensional $(0,1)$-matrix of order $n$. Then the following hold.

1. The number of $k$-multiplexes in the matrix $A$ is not greater than $\left(\frac{(k n) !}{k !^{n}}\right)^{d-1}$.

2. The number of true $k$-multiplexes is not greater than $\left(n \frac{(k n-2) !}{k !^{n-1}(k-2) !}\right)^{d}$.

3. The number of disconnected $k$-multiplexes that are a union of two $k$-multplexes of orders $n_{1}$ and $n_{2}\left(n_{1}+n_{2}=n\right)$ is not greater than $\left(\left(\begin{array}{c}n \\ n_{1}\end{array}\right) \frac{\left(k n_{1}\right) !\left(k n_{2}\right) !}{k !^{n}}\right)^{d}$.

4. Let $S$ be the minimal size of the support among the hyperplanes of the matrix $A$. If $k>S$ then there are no $k$-plexes on the support of $A$.

Proof. 1. As was mentioned before, if we fix the permutation in one coordinate of a multiplex then in each of the other $d-1$ coordinates we have a permutation of the multiset $I_{\{n, k\}}$.

2. If $K=\left\{\alpha^{1}, \ldots, \alpha^{k n}\right\}$ is a $k$-multiplex but not a $k$-plex then $K$ contains at least two identical elements. Without loss of generality, suppose that indices $\alpha^{1}$ and $\alpha^{2}$ are the same. For constructing a true $k$-multiplex $K$, in each of $d$ positions we have $n$ possibilities to choose a symbol for indices $\alpha^{1}$ and $\alpha^{2}$. The remaining $k n-2$ indices are defined by a permutation of the multiset of $k n-2$ elements in which each of $n-1$ symbols appears exactly $k$ times and the last symbol appears $k-2$ times.

3. If a $k$-multiplex $K=\left\{\alpha^{1}, \ldots, \alpha^{k n}\right\}$ is a union of two $k$-multiplexes of orders $n_{1}$ and $n_{2}$ then $K$ can be partitioned into two submultisets $K_{1}$ and $K_{2}$ of sizes $k n_{1}$ and $k n_{2}$ such that any two indices $\alpha^{i} \in K_{1}$ and $\alpha^{j} \in K_{2}$ differ at all positions. For constructing such a disconnected $k$-multiplex $K$, in each of $d$ positions we choose independently $n_{1}$ and $n_{2}$ symbols that appear in elements of $K_{1}$ and $K_{2}$ and then take permutations of the multisets $I_{\left\{n_{1}, k\right\}}$ and $I_{\left\{n_{2}, k\right\}}$.

4. By definition, each hyperplane of $A$ contains exactly $k$ different elements of a $k$-plex, so if the support of $A$ in one hyperplane is less than $k$ then $A$ has no $k$-plexes on it.

Using this lemma and Theorem 6 we prove for large $d$ that a typical $k$-multiplex in a $d$-iterated quasigroup is a connected indivisible $k$-plex.

Theorem 10. Let $G$ be a binary quasigroup of order $n$ and let $M\left(G^{[d]}\right)$ be the $(d+1)$ dimensional permutation matrix corresponding to the d-iterated quasigroup $G$. Then the ratio of the number of connected indivisible $k$-plexes to the number of $k$-multiplexes in $M\left(G^{[d]}\right)$ tends to one as $d$ tends to infinity. In other words, the ratio of the number of 
connected indivisible $k$-plexes to $\left(\frac{(k n) !}{k !^{n}}\right)^{d-1}$ tends to $c(G, k)$ as $d \rightarrow \infty$ with $d$ being such that $\operatorname{Per}_{k} M\left(G^{[d]}\right)>0$. Here $c(G, k)$ is the constant in Theorem 6 .

Proof. We prove that the ratios of the numbers of true $k$-multiplexes, divisible $k$-multiplexes, and disconnected $k$-multiplexes to the number of all $k$-multiplexes in $M\left(G^{[d]}\right.$ ) (if it is nonzero) tends to zero as $d$ tends to infinity, which implies the statement of the theorem.

By Theorem 6, there exists some constant $c \geqslant c(G, k)\left(\frac{(k n) !}{k ! n}\right)^{-1}>0$ such that if $\operatorname{Per}_{k} M\left(G^{[d]}\right)>0$ then

$$
\operatorname{Per}_{k} M\left(G^{[d]}\right) \geqslant c\left(\frac{(k n) !}{k !^{n}}\right)^{d}
$$

for all suitable $d$ starting from some $d_{0}$. So we have a lower bound on the number of all $k$-multiplexes in $M\left(G^{[d]}\right)$.

Let us find upper bounds on the numbers of true $k$-multiplexes, divisible $k$-multiplexes, and disconnected $k$-multiplexes in $M\left(G^{[d]}\right)$ and show that they are negligible with respect to the number of all $k$-multiplexes.

1. By Lemma 9, the number of true $k$-multiplexes in $M\left(G^{[d]}\right)$ is not greater than $\left(n \frac{(k n-2) !}{k !^{n-1}(k-2) !}\right)^{d}$. Therefore, the ratio of this number to the number of all $k$-multiplexes in $M\left(G^{[d]}\right)$ is not greater than $\frac{1}{c}\left(\frac{1}{k^{2}(k-1)(k n-1)}\right)^{d}$, which tends to zero as $d \rightarrow \infty$.

2. By Lemma 9, the number of $k$-multiplexes in $M\left(G^{[d]}\right)$ is not greater than $\left(\frac{(k n) !}{k !^{n}}\right)^{d}$. Consequently, the number of divisible $k$-multiplexes that are the union of a $k_{1}$-multiplex and a $k_{2}$-multiplex is not greater than $\left(\frac{\left(k_{1} n\right) !\left(k_{2} n\right) !}{k_{1} ! n k_{2} !^{n}}\right)^{d}$. Then the number of all divisible $k$-multiplexes is less than

$$
\sum_{k_{1}+k_{2}=k}\left(\frac{\left(k_{1} n\right) !\left(k_{2} n\right) !}{k_{1} !^{n} k_{2} !^{n}}\right)^{d} \leqslant k \max _{k_{1}+k_{2}=k}\left(\frac{\left(k_{1} n\right) !\left(k_{2} n\right) !}{k_{1} !^{n} k_{2} !^{n}}\right)^{d} .
$$

It is known that if $n \geqslant 2$ then

$$
\left(\begin{array}{c}
k n \\
k_{1} n
\end{array}\right)>\left(\begin{array}{c}
k \\
k_{1}
\end{array}\right)^{n}
$$

Indeed, if $k=k_{1}+k_{2}$ then the left-hand side of this inequality is the number of $k n$-tuples over the set of two symbols in which symbol $i$ appears exactly $k_{i} n$ times and the righthand side is the number of sets of $n k$-tuples over the same set of symbols in which symbol $i$ appears exactly $k_{i}$ times in each $k$-tuple.

Using this inequality and comparing the bound on divisible $k$-multiplexes with the number of all $k$-multiplexes we conclude that there exists a constant $0<\kappa<1$ such that their ratio is not greater than $\frac{k}{c} \kappa^{d}$ and tends to zero as $d \rightarrow \infty$. 
3. By Lemma 9, the number of disconnected $k$-multiplexes that are the union of $k$ multiplexes of orders $n_{1}$ and $n_{2}$ is not greater than $\left(\left(\begin{array}{c}n \\ n_{1}\end{array}\right) \frac{\left(k n_{1}\right) !\left(k n_{2}\right) !}{k !^{n}}\right)^{d}$. Then the number of all disconnected $k$-multiplexes is less than

$$
\sum_{n_{1}+n_{2}=n}\left(\left(\begin{array}{c}
n \\
n_{1}
\end{array}\right) \frac{\left(k n_{1}\right) !\left(k n_{2}\right) !}{k !^{n}}\right)^{d}<n \max _{n_{1}+n_{2}=n}\left(\left(\begin{array}{c}
n \\
n_{1}
\end{array}\right) \frac{\left(k n_{1}\right) !\left(k n_{2}\right) !}{k !^{n}}\right)^{d} .
$$

As is known, if $k \geqslant 2$ then

$$
\left(\begin{array}{c}
k n \\
k n_{1}
\end{array}\right)>\left(\begin{array}{c}
n \\
n_{1}
\end{array}\right)
$$

Using this inequality and comparing the bound on disconnected $k$-multiplexes with the number of all $k$-multiplexes we conclude that there exists a constant $0<\kappa<1$ such that their ratio is not greater than $\frac{n}{c} \kappa^{d}$ and tends to zero as $d \rightarrow \infty$.

Next, we specify Theorem 6 for plexes and multiplexes in iterated groups.

Theorem 11. Let $G$ be a group of order $n$ and let $M\left(G^{[d-1]}\right)$ be the d-dimensional permutation matrix of the iterated group $G$.

1. If $k$ is even and $d \geqslant 3$ then $M\left(G^{[d-1]}\right)$ has a $k$-multiplex. Moreover, there exists a constant $0<c(G, k) \leqslant 1$ such that

$$
\lim _{d \rightarrow \infty} \frac{\operatorname{Per}_{k} M\left(G^{[d-1]}\right)}{\left(\frac{(k n) !}{k !^{n}}\right)^{d-2}}=\lim _{d \rightarrow \infty} \frac{\operatorname{per}_{k} M\left(G^{[d-1]}\right)}{\left(\frac{(k n) !}{k !^{n}}\right)^{d-2}}=c(G, k),
$$

with there being a finite number of $d$ for which $\operatorname{per}_{k} M\left(G^{[d-1]}\right)$ is zero.

2. If $k$ is odd then there exists a constant $0<c(G, k) \leqslant 1$ such that

$$
\lim _{d \rightarrow \infty} \frac{\operatorname{Per}_{k} M\left(G^{[d-1]}\right)}{\left(\frac{(k n) !}{k ! n}\right)^{d-2}}=\lim _{d \rightarrow \infty} \frac{\operatorname{per}_{k} M\left(G^{[d-1]}\right)}{\left(\frac{(k n) !}{k ! n}\right)^{d-2}}=c(G, k),
$$

where the limits are taken over all $d$ for which $k$-permanents and $k$-multipermanents are nonzero.

Proof. 1. By Theorem 3, we have that $\operatorname{per}_{2} M\left(G^{[2]}\right)>0$. Using Lemma 8, we obtain that $\operatorname{Per}_{k} M\left(G^{[2]}\right)>0$ for all even $k$. Theorem 6 states that for all $d \geqslant 3$ the $k$-multipermanent of $M\left(G^{[d-1]}\right)$ is nonzero and there exists a constant $c(G, k)>0$ such that

$$
\lim _{d \rightarrow \infty} \frac{\operatorname{Per}_{k} M\left(G^{[d-1]}\right)}{\left(\frac{(k n) !}{k !^{n}}\right)^{d-2}}=c(G, k) .
$$

Since by Theorem 10, for large $d$ a typical $k$-multiplex in the iterated group $M\left(G^{[d-1]}\right)$ is a $k$-plex, we have that the $k$-permanent of $M\left(G^{[d-1]}\right)$ has the same limit and it equals to zero only for a finite number of $d$.

2. This clause is an immediate corollary of Theorem 6 and Theorem 10 . 
In conclusion, we note that if Rodney's conjecture is true then clause 1 of Theorem 11 holds for all quasigroups $G$, and if Ryser's conjecture is true then, in addition, this clause holds for all odd $k$ and quasigroups of odd orders $n$.

\section{Proof of Theorem 6}

The main idea of the proof of Theorem 6 is to show that the number of $k$-multiplexes in the multidimensional permutation matrix of an iterated quasigroup is a result of some Markov process.

Suppose $K=\left\{\alpha^{1}, \ldots, \alpha^{k n}\right\}$ is a $k$-multiplex in the permutation matrix $M\left(G^{[d-1]}\right)$ of order $n$. Consider the rectangular $k n \times d$ table $T=\left(t_{i, j}\right)$ such that $t_{i, j}=\alpha_{j}^{i}$. Note that the table $T$ has the following two properties:

1. Each column $T_{j}$ of $T$ is a permutation of the multiset $I_{\{n, k\}}$.

2. For columns $T_{j}$ of $T$ we have

$$
\left(\cdots\left(\left(T_{1} * T_{2}\right) * T_{3}\right) * \cdots * T_{d-1}\right) * T_{d}=E
$$

where $*$ is the operation of the quasigroup $G$.

Conversely, rows of every rectangular $k n \times d$ table $T$ satisfying these two properties comprise a $k$-multiplex in the $(d-1)$-iterated quasigroup $G$.

Permutations of rows of the table $T$ do not change the corresponding $k$-multiplex $K$, and if $K$ is a $k$-plex then it corresponds to exactly $(k n)$ ! tables $T$. By Lemma 9 , the number of true $k$-multiplexes is not greater than $\left(n \frac{(k n-2) !}{k !^{n-1}(k-2) !}\right)^{d}$, so to prove Theorem 6 it suffices to show that the number of such tables is asymptotically equal to $c(G, k)(k n) !\left(\frac{(k n) !}{k !^{n}}\right)^{d-2}$ for some constant $c(G, k)$. Note that $c(G, k)$ is not greater than 1 , because Lemma 9 says that any $d$-dimensional matrix of order $n$ has no more than $\left(\frac{(k n) !}{k !^{n}}\right)^{d-1} k$-multiplexes.

Given $m \geqslant 1$ and a $k n$-vector $U=\left(u_{1}, \ldots, u_{k n}\right)$, consider the set $\mathcal{T}_{U}(m)$ of all $k n \times m$ tables $T$ in which each column $T_{j}$ is a permutation of the multiset $I_{\{n, k\}}$ and such that

$$
\left(\cdots\left(\left(T_{1} * T_{2}\right) * T_{3}\right) * \cdots * T_{m-1}\right) * T_{m}=U .
$$

Let $W$ be a permutation of the multiset $I_{\{n, k\}}$ written as a column $k n$-vector. The number of such vectors is equal to the multinomial coefficient $\left(\begin{array}{c}k n \\ k \cdots k\end{array}\right)=\frac{(k n) !}{k !^{n}}$. For two column $k n$-vectors $U$ and $V$ over the set $I_{n}^{1}$ define $a_{U, V}$ to be the number of permutations $W$ such that $V * W=U$. By definition, we have that for given $U$ and $V$,

$$
\sum_{V^{\prime} \in I_{n}^{k n}} a_{U, V^{\prime}}=\sum_{U^{\prime} \in I_{n}^{k n}} a_{U^{\prime}, V}=\frac{(k n) !}{k !^{n}} .
$$


Note that if we extend a table $T$ from the set $\mathcal{T}_{V}(m-1)$ to the right by a permutation $W$ then we obtain a table from the set $\mathcal{T}_{U}(m)$ with $V * W=U$. For every $m$, the number $l_{U}(m)$ of tables from $\mathcal{T}_{U}(m)$ can be expressed as

$$
l_{U}(m)=\sum_{V \in I_{n}^{k n}} a_{U, V} l_{V}(m-1),
$$

where $a_{U, V}$ is independent of $m$.

If we suppose $L(m)$ to be a $n^{k n}$-vector of all $l_{U}(m)$, then we obtain

$$
L(m)=A(G) L(m-1)
$$

where $A(G)=\left(a_{U, V}\right)$ is a $(0,1)$-matrix of order $n^{k n}$. As was noted previously, the sum of the entries of the matrix $A(G)$ over each column and each row is equal to $\frac{(k n) !}{k !^{n}}$.

Next we normalize vectors $L(m)$ and the matrix $A(G)$ and put $X(m)=\left(\frac{(k n) !}{k !^{n}}\right)^{-m} L(m)$ and $B(G)=\left(\frac{(k n) !}{k !^{n}}\right)^{-1} A(G)$. Then we obtain the process

$$
X(m)=B(G) X(m-1)
$$

that is the Markov chain with a doubly stochastic transition matrix $B(G)$ (a nonnegative matrix with sums of entries over each row and each column equal to 1 ). For our purposes we are interested in the value of only one component of the vector $X(m)$, namely $x_{E}(m)$.

The present reasoning implies that Theorem 6 is a corollary of the following statement.

Proposition 12. Let $G$ be a quasigroup of order $n$. Suppose the matrix $B(G)$ and vectors $X(m)$ are as above and

$$
X(m)=B(G) X(m-1) .
$$

1. For all even $m$ we have $x_{E}(m)>0$. If for some odd $m^{\prime}$ we have $x_{E}\left(m^{\prime}\right)>0$ then $x_{E}(m)>0$ for all $m \geqslant m^{\prime}$.

2. For some constant $c=c(G)>0$ we have

$$
\lim _{m \rightarrow \infty} x_{E}(m)=c
$$

where all $m$ are such that $x_{E}(m)$ is nonzero.

To prove this statement we need to recall some concepts and theorems of the theory of Markov processes.

A Markov chain with a transition matrix $B$ (or the matrix itself) is called reducible if by simultaneous permutations of rows and columns the matrix $B$ can be put into a block upper triangular matrix of the form

$$
\left(\begin{array}{cc}
P & R \\
0 & S
\end{array}\right)
$$

where $P$ and $S$ are square matrices. A Markov chain is irreducible if it is not reducible. 
Let a matrix $B$ be irreducible. We define the period of state $i$ to be the greatest common divisor of all natural numbers $m$ for which the entry with index $(i, i)$ in the $m$-th power of $B$ is greater than zero. It is known that for an irreducible matrix $B$ each state $i$ has the same period that is called the period of $B$. If the period of a matrix $B$ is equal to 1 then the matrix $B$ is said to be aperiodic. The period of a Markov chain coincides with the period of its transition matrix.

The following theorem is the key result of the theory of Markov chains.

Theorem 13 (Ergodic theorem). A Markov chain

$$
X(m)=B X(m-1) ; x_{i}(0) \geqslant 0 ; \sum x_{i}(0)=1
$$

with a transition matrix $B$ is irreducible and aperiodic if and only if for each $i$ there exists $\lim _{m \rightarrow \infty} x_{i}(m)=c_{i}>0$ that does not depend on the initial state $X(0)$.

Now we are ready to prove Proposition 12.

Proof. Let $G$ be a quasigroup of order $n$ and $U, V \in I_{n}^{k n}$. Let $W$ be a permutation of the multiset $I_{\{n, k\}}$. Suppose $B(G)=b_{U, V}$ is a nonnegative matrix of order $n^{k n}$ such that $b_{U, V}$ is equal to $\frac{k !^{n}}{(k n) !}$ multiplied by the number of permutations $W$ for which $V * W=U$. Let us consider the Markov chain $X(m)=B(G) X(m-1), x_{E}(0)=1$, and $x_{U}(0)=0$ for all other vectors $U$.

We already know that the matrix $B(G)$ is doubly stochastic. It is not hard to prove that if a doubly stochastic matrix is reducible then by simultaneous permutations of rows and columns it can be put into a block-diagonal matrix in which each block is a doubly stochastic matrix of smaller order (a proof can be found, for example, in [4, p. 34]). If the matrix $B(G)$ is reducible then instead of the Markov chain with the matrix $B(G)$ we consider a process with the irreducible block containing entry $b_{E, E}$. So we may assume that $B(G)$ is an irreducible matrix.

The proof of Lemma 8 implies that the state $x_{E}(m)$ has period at most 2 , so matrix $B(G)$ also has period at most 2 . Moreover, $B(G)$ is aperiodic if for some odd $m$ we have $x_{E}(m)>0$.

1. If the matrix $B(G)$ is aperiodic, then by the ergodic theorem, there exists a constant $c=c(G)$ such that $\lim _{m \rightarrow \infty} x_{E}(m)=c$.

2. If the matrix $B(G)$ has period 2, then we consider the process $X(2 t)=B^{2}(G) X(2 t-$ 2), $x_{E}(0)=1$, and $x_{U}(0)=0$ for all other vectors $U$. The matrix $B^{2}(G)$ is aperiodic and we have $x_{E}(m)>0$ for all even $m$. So, by the ergodic theorem, there exists a constant $c=c(G)$ such that $\lim _{m \rightarrow \infty} x_{E}(m)=c$, where all $m$ are even.

Since we have now proved Proposition 12, Theorem 6 and all corollaries are also proved. 


\section{Computational results and concluding remarks}

At the end of this paper, we provide computational results on the numbers of transversals in the Cayley tables of iterated groups and quasigroups of small order, consider a generalization of Theorem 6 for partial transversals and plexes, and raise several problems.

It is not hard to see that latin hypercubes of orders 2 and 3 are unique up to equivalence and that they are the Cayley tables of the iterated groups $\mathbb{Z}_{2}$ and $\mathbb{Z}_{3}$. For order 4 there are plenty of non-equivalent multidimensional latin hypercubes but there are only two groups of order 4 , namely $\mathbb{Z}_{4}$ and $\mathbb{Z}_{2}^{2}$. The following table summarizes results from [7] and [9] on transversals in $d$-dimensional latin hypercubes that are the Cayley tables of $d$-iterated groups of orders $n \leqslant 4$.

\begin{tabular}{|c|c|c|}
\hline$G$ & $d$ even & $d$ odd \\
\hline $\mathbb{Z}_{2}$ & 0 & $2^{d-1}$ \\
\hline $\mathbb{Z}_{3}$ & $\frac{2}{3} \cdot 6^{d-1}-3^{d-2}$ & $\frac{2}{3} \cdot 6^{d-1}+3^{d-2}$ \\
\hline $\mathbb{Z}_{4}$ & 0 & $\frac{3}{8} \cdot 24^{d-1}+5 \cdot 8^{d-2}$ \\
\hline $\mathbb{Z}_{2}^{2}$ & $\frac{3}{8} \cdot 24^{d-1}-8^{d-2}$ & $\frac{3}{8} \cdot 24^{d-1}+5 \cdot 8^{d-2}$ \\
\hline
\end{tabular}

In this section, using the technique proposed in the proof of Theorem 6 , we count the number of transversals in the Cayley tables of the iterated group $\mathbb{Z}_{5}$ and of one iterated quasigroup of order 5 .

\subsection{Transversals in the iterated group $\mathbb{Z}_{5}$}

We start with the group $\mathbb{Z}_{5}$ whose Cayley table is as follows:

\begin{tabular}{l|lllll} 
& 1 & 2 & 3 & 4 & 5 \\
\hline 1 & 1 & 2 & 3 & 4 & 5 \\
2 & 2 & 3 & 4 & 5 & 1 \\
3 & 3 & 4 & 5 & 1 & 2 \\
4 & 4 & 5 & 1 & 2 & 3 \\
5 & 5 & 1 & 2 & 3 & 4
\end{tabular}

As before, let $l_{U}(d)$ be the number of tables $T$ from the set $\mathcal{T}_{U}(d)$ such that each of their columns $T_{j}$ is a permutation of the set of elements of $\mathbb{Z}_{5}$ and

$$
\left(\cdots\left(\left(T_{1} * T_{2}\right) * T_{3}\right) * \cdots\right) * T_{d}=U
$$

where $*$ is the $\mathbb{Z}_{5}$ group operation.

Note that if there exists a permutation $\sigma \in S_{5}$ such that $\left(u_{1}, \ldots, u_{5}\right)=\left(v_{\sigma(1)}, \ldots, v_{\sigma(5)}\right)$ then the numbers $l_{U}(d)$ and $l_{V}(d)$ are the same. Also, if vectors $U$ and $V$ satisfy $U * H=V$ where $H=(h, \ldots, h), h \in \mathbb{Z}_{5}$ then $l_{U}(d)=l_{V}(d)$. This fact is easy to prove by induction on $d$ using associativity of the group $\mathbb{Z}_{5}$ and the fact that for any permutation $W$ of the group $\mathbb{Z}_{5}$ and any $H=(h, \ldots, h)$ the vector $H * W$ is a permutation. We will say that vectors $U$ and $V$ are equivalent if they can be turned into each other by these two operations. 
Denote by $Z_{U}$ the equivalence class for vector $U$. By direct calculation, it can be checked that $l_{U}(d)$ is nonzero for some $d$ only if vector $U$ belongs to one of the following equivalence classes:

$$
Z_{11111}, Z_{12345}, Z_{11134}, Z_{11125}, Z_{11224}, Z_{11332} \text {. }
$$

Also, it can be verified that numbers $l_{U}(d)$ for $U$ from $Z_{11134}$ and $Z_{11125}$ satisfy similar relations, so they coincide and these two classes can be joined into one. The same is true for classes $Z_{11224}$ and $Z_{11332}$. For shortness, we introduce the following notations

$$
\begin{gathered}
y_{1}(d)=\sum_{U \in Z_{11111}} l_{U}(d) ; \quad y_{2}(d)=\sum_{U \in Z_{12345}} l_{U}(d) ; \\
y_{3}(d)=\sum_{U \in Z_{11134}, Z_{11125}} l_{U}(d) ; \quad y_{4}(d)=\sum_{U \in Z_{11224}, Z_{11332}} l_{U}(d) .
\end{gathered}
$$

Then the number of transversals in the $d$-dimensional latin hypercube that is the Cayley table of $d$-iterated group $\mathbb{Z}_{5}$ is equal to $\frac{1}{5 \cdot 120} y_{1}(d+1)$ or to $\frac{1}{120} y_{2}(d)$. By direct calculations, we establish that $y_{i}(d)$ satisfy the following recurrence relations:

$$
\begin{gathered}
\left(\begin{array}{l}
y_{1}(d) \\
y_{2}(d) \\
y_{3}(d) \\
y_{4}(d)
\end{array}\right)=\left(\begin{array}{cccc}
0 & 5 & 0 & 0 \\
120 & 15 & 30 & 20 \\
0 & 50 & 30 & 40 \\
0 & 50 & 60 & 60
\end{array}\right)\left(\begin{array}{l}
y_{1}(d-1) \\
y_{2}(d-1) \\
y_{3}(d-1) \\
y_{4}(d-1)
\end{array}\right) ; \\
y_{1}(0)=1 ; y_{2}(0)=y_{3}(0)=y_{4}(0)=0 .
\end{gathered}
$$

Note that these relations define a process with irreducible and aperiodic matrix $A$. So we have that for all $d \geqslant 1$ the number of transversals in the $d$-iterated group $\mathbb{Z}_{5}$ is greater than zero and that there exists a constant $c\left(\mathbb{Z}_{5}, 1\right)$ for which

$$
\lim _{d \rightarrow \infty} \frac{T\left(Q\left(\mathbb{Z}_{5}^{[d]}\right)\right)}{120^{d-1}}=c\left(\mathbb{Z}_{5}, 1\right)
$$

To find the limit constant $c\left(\mathbb{Z}_{5}, 1\right)$ we use the fact that $y_{1}(d)=\chi_{1} 120^{d}$ where $\chi_{1}$ is the first component of the eigenvector $\chi$ of the matrix $A$ corresponding to the largest eigenvalue $\lambda=120$ and normalized so that $\sum_{i=1}^{4} \chi_{i}=1$. Compute that

$$
\chi=\frac{1}{125}(1,24,40,60)^{T} .
$$

Thus $\chi_{1}=\frac{1}{125}$ and $c\left(\mathbb{Z}_{5}, 1\right)=\frac{120}{5} \chi_{1}=\frac{24}{125}$. Therefore, for the number of transversals in the $d$-dimensional $d$-iterated group $\mathbb{Z}_{5}$ and for the permanent of the corresponding $(d+1)$-dimensional permutation matrix we have

$$
\lim _{d \rightarrow \infty} \frac{T\left(Q\left(\mathbb{Z}_{5}^{[d]}\right)\right)}{120^{d-1}}=\lim _{d \rightarrow \infty} \frac{\operatorname{per} M\left(\mathbb{Z}_{5}^{[d]}\right)}{120^{d-1}}=\frac{24}{125} .
$$




\subsection{Transversals in the quasigroup of order 5}

Let us consider the quasigroup $G$ of order 5 defined by the following Cayley table:

\begin{tabular}{l|lllll} 
& 1 & 2 & 3 & 4 & 5 \\
\hline 1 & 1 & 2 & 3 & 4 & 5 \\
2 & 2 & 1 & 4 & 5 & 3 \\
3 & 3 & 4 & 5 & 1 & 2 \\
4 & 4 & 5 & 2 & 3 & 1 \\
5 & 5 & 3 & 1 & 2 & 4
\end{tabular}

We will use the same notations as before but now $Z_{U}$ will be the set of vectors $V$ that can be turned into the vector $U$ only via a permutation of components. By direct calculation, it can be checked that for all vectors $U$ the number $l_{U}(d)$ is not zero for certain $d$.

Analyzing the relations between numbers $l_{U}(d)$, we divide all vectors from the set $I_{5}^{5}$ into the following classes (different letters mean different values):

$$
H_{1}=\cup Z_{i i i i i} ; \quad H_{2}=Z_{12345} ; \quad H_{3}=\bigcup Z_{222 i j} \cup Z_{i i i 2 j} ;
$$

$H_{4}=\cup Z_{i i i j k}$, where $i, j, k \neq 2 ; \quad H_{5}=\bigcup Z_{222 i i} \cup Z_{i i i 22} ; \quad H_{6}=\cup Z_{i i i j j}$, where $i, j \neq 2$;

$$
H_{7}=\cup Z_{i i 2 j k} ; \quad H_{8}=\cup Z_{i i j k l}, \text { where } i, j, k, l \neq 2 ; \quad H_{9}=\cup Z_{22 i j k} ;
$$

$H_{10}=\cup Z_{22 i i k} ; \quad H_{11}=\cup Z_{i i j j k}$, where $i, j, k \neq 2 ; \quad H_{12}=\cup Z_{i i j j 2} ; \quad H_{13}=\cup Z_{i i i i j}$.

Put $y_{i}(d)=\sum_{U \in H_{i}} l_{U}(d)$ for all $i=1, \ldots, 13$ and denote $Y(d)=\left(y_{1}(d), \ldots, y_{13}(d)\right)^{T}$. By calculations, we found that $Y(d)$ satisfies the process $Y(d)=A \cdot Y(d-1)$ with the matrix

$$
A=\left(\begin{array}{ccccccccccccc}
0 & 5 & 0 & 0 & 0 & 0 & 0 & 0 & 0 & 0 & 0 & 0 & 0 \\
120 & 3 & 6 & 12 & 12 & 0 & 2 & 2 & 8 & 0 & 4 & 12 & 0 \\
0 & 18 & 18 & 18 & 0 & 0 & 12 & 18 & 18 & 12 & 12 & 24 & 0 \\
0 & 24 & 12 & 12 & 0 & 0 & 8 & 6 & 6 & 12 & 12 & 8 & 0 \\
0 & 8 & 0 & 0 & 0 & 0 & 4 & 0 & 0 & 8 & 8 & 0 & 0 \\
0 & 0 & 0 & 0 & 0 & 0 & 6 & 6 & 6 & 8 & 8 & 8 & 0 \\
0 & 12 & 24 & 24 & 36 & 36 & 30 & 24 & 24 & 28 & 28 & 16 & 72 \\
0 & 4 & 12 & 6 & 0 & 12 & 8 & 12 & 6 & 12 & 8 & 8 & 24 \\
0 & 16 & 12 & 6 & 0 & 12 & 8 & 6 & 0 & 16 & 12 & 0 & 24 \\
0 & 0 & 12 & 18 & 36 & 24 & 14 & 18 & 24 & 4 & 8 & 24 & 0 \\
0 & 12 & 12 & 18 & 36 & 24 & 14 & 12 & 18 & 8 & 12 & 16 & 0 \\
0 & 18 & 12 & 6 & 0 & 12 & 4 & 6 & 0 & 12 & 8 & 4 & 0 \\
0 & 0 & 0 & 0 & 0 & 0 & 10 & 10 & 10 & 0 & 0 & 0 & 0
\end{array}\right) .
$$

The eigenvector $\chi$ of the matrix $A$ corresponding to the largest eigenvalue $\lambda=120$ with $\sum_{i=1}^{13} \chi_{i}=1$ is

$$
\chi=\frac{1}{625}(1,24,72,48,16,24,144,48,48,72,72,36,20)^{T} .
$$


So the limit constant $c(G, 1)$ for the number of transversals in the $d$-dimensional $d$ iterated quasigroup $G$ and for the permanent of the corresponding $(d+1)$-dimensional permutation matrix is $c(G, 1)=\frac{24}{625}$.

\subsection{Remarks and open questions}

First of all, we would like to note that the technique proposed in this paper can be used not only to count transversals in iterated quasigroups of order 5 but to estimate the number of transversals and $k$-plexes in other small-ordered iterated quasigroups and to find these numbers exactly for all small dimensions.

Secondly, we note that $d$-iterated quasigroups obtained from isotopic binary quasigroups can be non-isotopic and can have different numbers of transversals and plexes. For example, there exists a binary quasigroup $G$ of order 4 isotopic to the group $\mathbb{Z}_{4}$ but in contrast to the $d$-iterated group $\mathbb{Z}_{4}$ the $d$-iterated quasigroup $G$ has transversals for all $d \geqslant 3$.

Imitating the proof of Theorem 6, we can obtain the similar theorem for partial structures in iterated quasigroups. A partial diagonal of length $l$ in a $d$-dimensional matrix of order $n$ is a set of $l$ indices such that each hyperplane contains no more than one index from the set. A partial transversal in a $d$-dimensional latin hypercube $Q$ of order $n$ is a set of indices corresponding to a unity partial diagonal in the $(d+1)$-dimensional matrix $M(Q)$.

A partial $k$-multidiagonal of length $l$ in a multidimensional matrix is a multiset of $k l$ indices such that there are either $k$ or zero indices in each hyperplane. A partial $k$ multidiagonal in a multidimensional permutation matrix corresponds to a partial multiplex in a latin hypercube.

For a $d$-dimensional $(0,1)$-matrix $A$ of order $n$ let us denote by $P_{l, k}(A)$ the number of partial $k$-multidiagonals of length $l$ over the support of $M$. Using the same method as in the proof of Theorem 6 , we can obtain the following statement.

Proposition 14. Let $G$ be a binary quasigroup of order $n$ and let $M\left(G^{[d-1]}\right)$ be the $d$ dimensional permutation matrix of the $(d-1)$-iterated quasigroup $G$.

1. For all even $d$ and for all $l \leqslant n$ the permutation matrix $M\left(G^{[d-1]}\right)$ has a positive partial $k$-multidiagonal of length $l$. If for some odd $d^{\prime}$ we have that $P_{l, k}\left(M\left(G^{\left[d^{\prime}-1\right]}\right)\right)$ is positive then $P_{l, k}\left(M\left(G^{[d-1]}\right)\right)$ is greater than zero for all $d \geqslant d^{\prime}$.

2. There exists a constant $c(G, k, l)>0$ such that

$$
\lim _{d \rightarrow \infty} \frac{P_{l, k}\left(M\left(G^{[d-1]}\right)\right)}{\left(\left(\begin{array}{l}
n \\
l
\end{array}\right) \frac{(k l) !}{k ! !^{l}}\right)^{d-2}}=c(G, k, l),
$$

where the limit is taken on the set of all $d$ for which $M\left(G^{[d-1]}\right)$ contains a positive partial $k$-multidiagonal of length $l$. 
In conclusion, we raise several questions and open problems inspired by the obtained results:

1. How can the limit constants $c(G, k)$ for the number of $k$-plexes be estimated in general and for explicit groups and quasigroups $G$ ?

2. How are the limit constants $c(G, k)$ for the number of $k$-plexes in a given iterated quasigroup $G$ related to each other for different $k$ ?

3. For odd $n$ or $d$, does some $d$-iterated group always have the maximum number of transversals (and possibly $k$-plexes and $k$-multiplexes) among all iterated quasigroups of order $n$ ?

4. Suppose $k \leqslant n^{d-1}$ and a latin hypercube $Q$ has a $k$-multiplex. Is it true that $Q$ has a $k$-plex?

\section{References}

[1] C. J. Colbourn and J. H. Dinitz (eds). Handbook of combinatorial designs (2nd ed.). Chapman \& Hall/CRC, Boca Raton, 2007.

[2] S. Eberhard. More on additive triples of bijections. arXiv:1704.02407.

[3] R. Glebov, Z. Luria. On the maximum number of latin transversals. J. Combin. Theory A, 141:136-146, 2016.

[4] H. Minc. Permanents. Encyclopedia of Mathematics and Its Applications, Vol 6, Addison-Wesley, Reading, Mass., 1978.

[5] K. Pula. A generalization of plexes of latin squares. Discrete Math., 311:577-581, 2011.

[6] H. J. Ryser. Neuere Probleme der Kombinatorik. Vortrage über Kombinatorik Oberwolfach, 24-29 Juli. 69-91, 1967.

[7] A. A. Taranenko. Permanents of multidimensional matrices: Properties and applications. J. Appl. Ind. Math., 10(4):567-604, 2016. (Translation from Russian. Original text published in Diskretn. Anal. Issled. Oper., 23(4):35-101, 2016)

[8] A. A. Taranenko. Multidimensional permanents and an upper bound on the number of transversals in latin squares. J. Combin. Des., 23:305-320, 2015.

[9] A. A. Taranenko. Transversals in completely reducible multiary quasigroups and in multiary quasigroups of order 4. Discrete Math., 341:405-420, 2018.

[10] M. Vaughan-Lee, I. M. Wanless. Latin squares and the Hall-Paige conjecture. Bull. London Math. Soc., 35:1-5, 2003.

[11] I. M. Wanless. Transversals in latin squares: a survey. In Surveys in Combinatorics 2011, volume 392 of London Mathematical Society Lecture Note Series, pages 403-437. Cambridge University Press, 2011. 